Beiträge zur Chemie des Schwefels $89^{1}$

\section{Über die Darstellung neuer Thiosilanole, Thiolisanolate sowie eines neuen Silanolats}

\author{
F. FeHÉr und H. Goller ${ }^{2}$
}

Institut für Anorganische Chemie der Universität Köln

(Z. Naturforschg. 22 b, 1223 [1967]; eingegangen am 20. Juli 1967)

Im Rahmen unserer Arbeiten über kettenförmige Schwefelverbindungen wurden als Ausgangsprodukte der Synthese von Silyl- und Siloxysulfanen ${ }^{3}$ erstmalig dargestellt: Tribenzylthiosilanol, Tri-p-tolylthiosilanol, Natriumtribenzylthiosilanolat, Natriumtri- $p$-tolylthiosilanolat und Natriumtri-p-tolylsilanolat.

I. Tribenzylthiosilanol wurde in Benzol bei Zimmertemperatur durch Umsetzung von Tribenzylchlorsilan ${ }^{4}$ mit Schwefelwasserstoff in Gegenwart von Triäthylamin gewonnen. Die Reinigung erfolgte durch Umkristallisieren aus einem Schwefelkohlenstoff-Petroläther-Gemisch.

Ausbeute 74\%; Schmp. $128-129^{\circ}$.

Analyse von $\left(\mathrm{C}_{6} \mathrm{H}_{5} \mathrm{CH}_{2}\right)_{3} \mathrm{SiSH}(334,6)$

Ber. S 9,58 Si 8,40 ,

Gef. S 9,68 Si 8,42.

Tri-p-tolylthiosilanol konnte durch 14-stdg. Kochen äquimolarer Mengen Tri- $p$-tolylsilan ${ }^{5}$ und Schwefel in Decalin erhalten werden. Unter Zusatz von Kohle wurde das Rohprodukt aus Petroläther umkristallisiert. Das Verfahren entspricht weitgehend dem der Darstellung von Triphenylthiosilanol ${ }^{6}$.

Ausbeute 52\%; Schmp. $106^{\circ}$.

Analyse von $\left(p-\mathrm{CH}_{3} \mathrm{C}_{6} \mathrm{H}_{4}\right)_{3} \mathrm{SiSH}(334,6)$

Ber. S 9,58 Si 8,40,

Gef. S 9,57 Si 8,31.

II. Natriumtribenzylthiosilanolat und Natriumtri-ptolylthiosilanolat wurden aus den entsprechenden Thiosilanolen durch Salzbildung mittels des sehr reaktionsfähigen Natriumhydrids hergestellt. Bei dieser Umsetzung reagieren der in der SH-Bindung positiv und in

1 87. u. 88. Mitt.: F. Fehér u. B. Degen, Angew. Chem. 79, 689 u. 690 [1967].

2 H. Goller, Dissertation, Universität Köln 1967.

3 90. u. 91. Mitt.: F. FeHÉR u. H. GolLER, Z. Naturforschg. 22 b, 1224, 1225 [1967].

4 G. Martin u. F. S. Kipping, J. chem. Soc. [London] 95, 302 [1909].

5 R. A. Benkeser u. F. J. Riel, J. Amer. chem. Soc. 73, 3472 [1951].

6 R. Calas, N. Duffaut u. B. Martel, Bull. Soc. chim. France 1961, 886. der Hydrid-Bindung negativ polarisierte Wasserstoff unter Bildung der $\mathrm{H}_{2}$-Molekel.

Zur Darstellung des Natriumtribenzylthiosilanolats wurde eine Lösung von Tribenzylthiosilanol in Benzol während 30 Min. tropfenweise unter Rühren und Durchleiten von Stickstoff zu einer Suspension von Natriumhydrid (5-facher Uberschuß) in Benzol gegeben. Nach weiterem 1-stdg. Rühren wurde kurz zum Sieden erhitzt, überschüssiges Natriumhydrid abgesaugt und das Lösungsmittel im Vakuum abgezogen. Auskochen des Rückstandes mit Ligroin $\left(60-90^{\circ}\right)$ ergab ein reines Produkt.

Ausbeute 92 Prozent.

Gehaltsbestimmung von $\left(\mathrm{C}_{6} \mathrm{H}_{5} \mathrm{CH}_{2}\right)_{3} \mathrm{SiSNa}(356,5)$ $100,0 \%$, ermittelt durch Titration der methanolischen Lösung mit $n / 10-\mathrm{HCl}$.

Natriumtribenzylthiosilanolat ist stark hygroskopisch und in Benzol, Toluol, Tetrahydrofuran und Dioxan löslich.

Die Darstellung von Natriumtri-p-tolylthiosilanolat erfolgte in Toluol, die Reinigung durch Auskochen mit Benzol. Im übrigen wurde wie oben verfahren.

Ausbeute 81 Prozent.

Gehaltsbestimmung von $\left(p-\mathrm{CH}_{3} \mathrm{C}_{6} \mathrm{H}_{4}\right)_{3} \mathrm{SiSNa}(356,5)$ $93,0 \%$, ermittelt wie bei Natriumtribenzylthiosilanolat.

Natriumtri-p-tolylthiosilanolat ist sehr schwer löslich in Benzol, Äther u. a. Eine mittlere Löslichkeit besteht in siedendem Toluol und Tetrahydrofuran.

III. Das bisher ebenfalls nicht bekannte Natriumtrip-tolylsilanolat erhielten wir - unter Anwendung der bei Natriumtribenzylthiosilanolat beschriebenen $\mathrm{Ar}$ beitstechnik - aus Tri-p-tolylsilanol ${ }^{7}$ und Natriumhydrid.

Ausbeute 94 Prozent.

Gehaltsbestimmung von $\left(p-\mathrm{CH}_{3} \mathrm{C}_{6} \mathrm{H}_{4}\right)_{3} \mathrm{SiONa}(340,5)$ $99,9 \%$, ermittelt wie bei Natriumtribenzylthiosilanolat.

IV. Auch zur Darstellung der bereits bekannten Verbindungen Natriumtriphenylthiosilanolat ${ }^{8}$ und $\mathrm{Na}$ triumtriphenylsilanolat ${ }^{9,10}$ wurde von uns Natriumhydrid verwendet. Die Salze waren nach diesem Verfahren leicht in reiner Form aus Triphenylthiosilanol ${ }^{6}$ bzw. Triphenylsilanol ${ }^{11}$ zugänglich.

7 Tri-p-tolylsilanol wurde durch Hydrolyse von Tri-p-tolylchlorsilan dargestellt. Tri-p-tolylchlorsilan erhielten wir aus $p$-Tolyllithium und Di- $p$-tolyldichlorsilan und die letztere Verbindung aus Siliciumtetrachlorid und $p$-Tolyllithium.

8 L. Birkofer, A. Ritter u. H. Goller, Chem. Ber. 96, 3289 [1963].

9 W. Schlenk, J. Renning u. G. Racky, Ber. dtsch. chem. Ges. 44, 1178 [1911].

10 F. Hyde, O. K. Johannson, W. H. Daudt, R. F. Fleming, H. B. Laudenslager u. M. P. Roche, J. Amer. chem. Soc. 75 , 5615 [1953].

11 Triphenylsilanol wurde durch Hydrolyse des aus Lithiumphenyl und Diphenyldichlorsilan gewonnenen Triphenylchlorsilans dargestellt. 